\title{
CARACTERÍSTICAS ANTROPOMÉTRICAS DE ADERENTES E DESISTENTES DE TREINAMENTO DE FORÇA EM ACADEMIA
}

\author{
ANTHROPOMETRIC CHARACTERISTICS OF ADHERENTS AND DROPOUTS \\ OF STRENGTH TRAINING
}

\author{
Alexandro Andradea , Fábio Hech Dominski ${ }^{b}$, Carla Maria Lizc ${ }^{c}$ Clara Knierim Correia ${ }^{d}$ \\ aalexandro.andrade@udesc.br, bfabiohdominski@hotmail.com, 'ccarla.maria.liz@gmail.com, dclara.kc@hotmail.com \\ Universidade do Estado de Santa Catarina - Florianópolis (SC), Brasil
}

Data de recebimento do artigo: 31/03/2016

Data de aceite do artigo: 16/08/2016

\section{RESUMO}

Objetivo: Comparar o perfil antropométrico de aderentes e desistentes de treinamento de força (TF) de diferentes sexos, idades e estados civis. Método: Participaram do estudo 54 praticantes de TF, com média de idade de 30 anos, que foram caracterizados quanto a sexo, idade e estado civil e quanto aos indicadores antropométricos (peso corporal, estatura, razão cintura-quadril, índice de massa corporal e percentual de gordura). Os dados foram tratados com estatística descritiva e inferencial. Resultados: Aderentes e desistentes do TF não se diferenciaram significativamente com relação aos índices antropométricos. Foi verificado que os desistentes do TF com parceiro apresentaram maior percentual de gordura e massa gorda quando comparados aos sem parceiro. Aderentes e desistentes acima de 30 anos apresentaram maior percentual de gordura e massa gorda do que os de até 30 anos. Conclusão: Aderentes e desistentes não se diferenciaram significativamente com relação aos índices antropométricos.

Palavras-chave: Treinamento de resistência; antropometria; academias de ginástica.

\section{ABSTRACT}

Objective: The aim of this study was to compare the anthropometric profile of adherents and dropouts of strength training with different gender, age and civil status. Methods: 54 practitioners of strength training participated in this study, with average age 30 years. Participants were characterized according to gender, age and civil status and the anthropometric indicators (body weight, height, waist-hip ratio, body mass index and body fat). Data was analized by descriptive and inferential statistics. Results: Adherents and dropouts do not differ significantly from anthropometric indicators. Dropouts with partner, have a higher body fat and fat mass compared to those without partner. Adherents and dropouts over 30 have a higher body fat and fat mass than 30 years. Conclusion: Adherents and dropouts do not differ significantly from anthropometric indicators.

Keywords: Resistance training; anthropometry; fitness centers. 


\section{Introdução}

A busca pela prática regular de exercícios físicos pela populaçáo tem incentivado o crescimento do setor de academias de musculação e ginástica no Brasil. Segundo dados da International Health, Racquet \& Sportsclub Association ${ }^{1}$, o número de academias no Brasil passou de 7,5 mil, em 2006, para 30.767, em 2014. Também foi observado o aumento no número de alunos desse ambiente - de 3,5 milhóes, em 2006, para 7 milhóes, em 2014.

Uma das modalidades de exercício físico mais praticadas nas academias é o treinamento de força (TF $)^{2}$. Estudos mostram que ele gera benefícios como redução de gordura corporal e aumento de massa muscular ${ }^{3}$. Tais benefícios são influenciados, além de pela prática regular do TF, pela alimentação balanceada ${ }^{4,5}$.

Cientes dos benefícios estéticos proporcionados pelo TF, homens e mulheres, de diferentes idades e estados civis, têm buscado a prática em academias ${ }^{6}$. Por outro lado, muitos iniciantes no TF almejam esses resultados estéticos em um curto espaço de tempo e geralmente não seguem de maneira regular o programa de TF nem alteram seus hábitos alimentares após o início da prática. Isso os leva à desistência da prática sem que tenham atingido os resultados estéticos esperados.

Os resultados estéticos podem ser verificados por meio da avaliação da composição corporal. Esse é um importante instrumento a ser utilizado por profissionais atuantes em academias para mensurar os efeitos sobre seu aluno do programa de TF proposto. A detecçáo do excesso de peso e o conhecimento do aspecto de sua distribuição exige a avaliação dessa composição, e a antropometria é o método mais utilizado - ela mensura a medida do tamanho corporal e de suas proporçôes pelos parâmetros de peso, estatura, pregas cutâneas e circunferências ${ }^{7}$.

O percentual de gordura pode ser obtido a partir de uma técnica antropométrica, amplamente utilizada, da mensuração de dobras cutâneas, cujo resultado auferido não difere significativamente do percentual de gordura pela pesagem hidrostática, que é tida como critério para validação de outros métodos ${ }^{8}$. $\mathrm{O}$ índice de massa corporal (IMC) é normalmente utilizado na avaliação do estado nutricional e é importante na avaliaçáo do risco de mortalidade. O fator limitante à aplicação do IMC é que ele não fornece informaçóes relacionadas com a composiçáo corporal: indivíduos com elevada quantidade de massa muscular podem apresentar elevado IMC, mesmo que a gordura corporal não seja excessiva? .

Para mensuração de obesidade abdominal pode ser utilizada a razão cinturaquadril (RCQ), que se relaciona com o aumento do risco de infarto do miocárdio, de acidente vascular encefálico e de morte prematura, porém recentes estudos apontam a perda de sua sensibilidade como indicador de risco, pois o quadril inclui a gordura subcutânea pélvica, a massa muscular e o tamanho do osso pélvico horizontal ${ }^{10}$.

Considerando isso, o objetivo deste estudo foi comparar o perfil antropométrico de aderentes e desistentes de TF de diferentes sexos, idades e estados civis.

\section{Métodos}

\section{Caracterização da pesquisa}

Trata-se de um estudo descritivo de campo transversal. A pesquisa foi aprovada pelo Comitê de Ética em Pesquisas com Seres Humanos da Universidade do Estado de Santa Catarina (Parecer 159198/2012).

\section{Participantes}

Participaram do estudo 54 praticantes de TF, com média de idade de 30 anos $( \pm 8)$ (mínima 18 , máxima 58 anos), sendo 32 homens (59,3\%) e 22 mulheres $(40,7 \%)$. Esses praticantes foram selecionados de maneira não probabilística intencional, seguindo o critério de voluntariado em uma academia de musculação de Florianópolis (SC).

Os participantes foram divididos em dois grupos: 1) aderentes - praticantes de TF há mais de 6 meses $(\mathrm{n}=25$ $(46,3 \%))$, com média de idade de 29,28 anos $( \pm 8,54)$ e tempo de prática de TF de 41,7 meses $( \pm 42,08) ; 2)$ desistentes - não praticantes de TF $(\mathrm{n}=29(53,7 \%))$, com média de idade de 30,62 anos $( \pm 9,25)$.

\section{Procedimentos e instrumentos}

A coleta de dados ocorreu em outubro de 2013. Os aderentes de TF que estavam presentes na academia no momento da coleta receberam informaçóes detalhadas sobre o estudo e concordaram em participar mediante assinatura do Termo de Consentimento Livre e Esclarecido e preenchimento do questionário do estudo.

O grupo de desistentes do TF foi localizado no cadastro de ex-usuários da academia com a autorizaçáo do gestor. O critério estabelecido para formação desse grupo foi que eles não praticavam TF há mais de seis meses. $\mathrm{O}$ agendamento para realização da avaliação física e preenchimento do questionário foi realizado via telefone.

Todos os participantes foram caracterizados quanto a sexo, idade e estado civil, e todas as avaliaçôes antropométricas foram realizadas em uma sala destinada para tal na própria academia. 
Com relação aos indicadores antropométricos, foram realizadas as seguintes medidas: peso corporal $(\mathrm{kg})$, estatura (m), índice de massa corporal (IMC), dobras cutâneas $(\mathrm{mm})$, massa magra (MM em kg) e massa gorda (MG em kg).

O peso corporal dos praticantes de TF foi obtido utilizando-se uma balança mecânica da marca Welmy, com resolução de $0,1 \mathrm{~kg}$. A estatura foi medida pela régua antropométrica com escala de 2,00 m fixada à balança. Obtidos os valores de peso corporal e estatura, foi calculado o IMC, por meio da divisão do peso corporal pelo quadrado da estatura $\left(\mathrm{kg} / \mathrm{m}^{2}\right)$.

Utilizou-se fita métrica metálica e não flexível de marca Cescorf para mensuração das circunferências da cintura e do quadril e para posterior cálculo da RCQ. Para aferição das dobras cutâneas foi utilizado um adipômetro científico de marca Cescorf, modelo Top Tec, com precisão de $1 \mathrm{~mm}$. Foram aferidas as dobras cutâneas subescapular, tricipital e suprailíaca e a panturrilha. Seguiu-se o protocolo descrito por Petroski ${ }^{11}$ para a estimativa da densidade corporal. A estimativa de percentual de gordura (\% G) foi calculada pela equaçáo de Siri ${ }^{12}$.

\section{Análise estatística}

Os dados foram analisados no programa Statistic Package for the Social Sciences (SPSS), versão 20.0. Utilizou-se de estatística descritiva padrão para verificação de distribuição dos dados, distribuição das frequências, percentuais, máximos e mínimos, análises das tendências centrais e dispersão dos dados. Para comparar as características antropométricas entre aderentes e desistentes do TF e entre diferentes faixas etárias, sexos e estados civis, empregou-se o teste U, de Mann Whitney. A correlaçáo das características antropométricas em funçáo da idade foi verificada por meio do teste Rho de Spearman (p). Para todas as análises adotou-se $\alpha$ de 0,05 ( $<<0,05)$.

\section{Resultados}

Aderentes e desistentes do TF não se diferenciaram significativamente com relação às variáveis antropométricas: peso $(\mathrm{p}=0,35)$, percentual de gordura $(\mathrm{p}=0,10)$, RCQ $(p=0,28)$ e massa magra $(p=0,65)$. Por outro lado, pode-se verificar a tendência de maiores médias de IMC $(p=0,06)$ e massa gorda $(p=0,05)$ entre os desistentes (Tabela 1$)$.

Com relação às diferenças antropométricas entre homens e mulheres aderentes e desistentes do TF, em ambos os grupos os homens apresentaram maiores peso, RCQ e massa magra do que as mulheres. Por outro lado, em ambos os grupos as mulheres apresentaram maior percentual de gordura em comparação aos homens. Somente no grupo de aderentes os homens apresentaram maior IMC do que as mulheres (Tabela 1).

Quando comparadas as médias das variáveis antropométricas de homens aderentes e desistentes do TF, foi verificado que os desistentes apresentaram maior percentual de gordura do que os aderentes $(\mathrm{p}=0,04)$. Ainda, as mulheres desistentes do TF apresentaram significativamente maiores peso corporal $(\mathrm{p}=0,02)$, IMC $(\mathrm{p}=0,01)$ e massa magra $(\mathrm{p}=0,02)$ do que as mulheres aderentes ao TF.

Tabela 1 - Análise comparativa das características antropométricas de homens e mulheres aderentes e desistentes de TF ( $\dot{x} / \pm$ ).

\begin{tabular}{|c|c|c|c|c|c|}
\hline & \multirow{2}{*}{ Geral } & \multicolumn{2}{|c|}{ Aderentes } & \multicolumn{2}{|c|}{ Desistentes } \\
\hline & & Homens & Mulheres & Homens & Mulheres \\
\hline \multirow{3}{*}{ Peso $(\mathrm{Kg})$} & \multirow{3}{*}{$70,25 \pm 12,97$} & \multicolumn{2}{|c|}{$67,84, \pm 13,32$} & \multicolumn{2}{|c|}{$72,32 \pm 12,53$} \\
\hline & & $75,97 \pm 10,34$ & $55,66 \pm 5,65$ & $77,10 \pm 12,44$ & $65,56 \pm 9,45$ \\
\hline & & \multicolumn{2}{|c|}{$\mathrm{p}<0,01$} & \multicolumn{2}{|c|}{$\mathrm{p}=0,01$} \\
\hline \multirow{3}{*}{$\% \mathrm{G}$} & \multirow{3}{*}{$20,76 \pm 7,25$} & \multicolumn{2}{|c|}{$18,94 \pm 6,55$} & \multicolumn{2}{|c|}{$22,39 \pm 7,56$} \\
\hline & & $15,38 \pm 5,45$ & $24,29 \pm 3,90$ & $19,37 \pm 6,26$ & $27,06 \pm 7,23$ \\
\hline & & \multicolumn{2}{|c|}{$\mathrm{p}<0,01$} & \multicolumn{2}{|c|}{$\mathrm{p}=0,01$} \\
\hline \multirow{3}{*}{ RCQ } & \multirow{3}{*}{$0,78 \pm 0,69$} & \multicolumn{2}{|c|}{$0,77 \pm 0,06$} & \multicolumn{2}{|c|}{$0,79 \pm 0,06$} \\
\hline & & $0,82 \pm 0,50$ & $0,71 \pm 0,025$ & $0,83 \pm 0,05$ & $0,73 \pm 0,05$ \\
\hline & & \multicolumn{2}{|c|}{$\mathrm{p}<0,01$} & \multicolumn{2}{|c|}{$\mathrm{p}<0,01$} \\
\hline \multirow{3}{*}{ IMC } & \multirow{3}{*}{$24,25 \pm 3,29$} & \multicolumn{2}{|c|}{$23,32 \pm 2,84$} & \multicolumn{2}{|c|}{$24,92 \pm 3,59$} \\
\hline & & $24,50 \pm 2,91$ & $21,56 \pm 1,61$ & $25,62 \pm 3,58$ & $24,23 \pm 3,34$ \\
\hline & & \multicolumn{2}{|c|}{$\mathrm{p}=0,01$} & \multicolumn{2}{|c|}{$\mathrm{p}=0,28$} \\
\hline
\end{tabular}

continua... 
Tabela 1: Continuação

\begin{tabular}{|c|c|c|c|c|c|}
\hline & \multirow{2}{*}{ Geral } & \multicolumn{2}{|c|}{ Aderentes } & \multicolumn{2}{|c|}{ Desistentes } \\
\hline & & Homens & Mulheres & Homens & Mulheres \\
\hline \multirow{3}{*}{$\mathrm{MM}(\mathrm{Kg})$} & \multirow{3}{*}{$55,64 \pm 11,18$} & \multicolumn{2}{|c|}{$55,39 \pm 12,91$} & \multicolumn{2}{|c|}{$55,57 \pm 9,66$} \\
\hline & & $64,15 \pm 8,60$ & $42,24 \pm 3,06$ & $61,52 \pm 6,11$ & $47,13 \pm 7,17$ \\
\hline & & \multicolumn{2}{|c|}{$\mathrm{p}<0,01$} & \multicolumn{2}{|c|}{$\mathrm{p}<0,01$} \\
\hline \multirow{3}{*}{ MG (Kg) } & \multirow{3}{*}{$14,62 \pm 6,23$} & \multicolumn{2}{|c|}{$12,56 \pm 4,36$} & \multicolumn{2}{|c|}{$16,12 \pm 7,01$} \\
\hline & & $11,81 \pm 5,00$ & $13,70 \pm 3,07$ & $15,58 \pm 7,31$ & $17,82 \pm 6,88$ \\
\hline & & \multicolumn{2}{|c|}{$\mathrm{p}=0,13$} & \multicolumn{2}{|c|}{$\mathrm{p}=0,35$} \\
\hline
\end{tabular}

Legenda: \% G = percentual de gordura; $\mathrm{RCQ}$ = razão cinturaquadril; $\mathrm{IMC}=$ índice de massa corporal; $\mathrm{MM}$ = massa magra; $\mathrm{MG}=$ massa gorda.

Com relação ao estado civil de aderentes e desistentes de TF, foi verificado que os desistentes do TF com parceiro apresentaram maior percentual de gordura e massa gorda quando comparados aos sem parceiro (Tabela 2).

Não foram verificadas diferenças significativas nas variáveis antropométricas entre os aderentes e desistentes com parceiro ou mesmo entre os aderentes e desistentes sem parceiro $(p>0,05)$.
Tabela 2 - Análise comparativa das características antropométricas de aderentes e desistentes de TF com parceiro e sem parceiro $(\dot{\mathrm{x}} / \pm)$.

\begin{tabular}{|c|c|c|c|c|}
\hline & \multicolumn{2}{|c|}{ Aderentes } & \multicolumn{2}{|c|}{ Desistentes } \\
\hline & Com parceiro & Sem parceiro & Com parceiro & Sem parceiro \\
\hline \multirow{2}{*}{ Peso $(\mathrm{Kg})$} & $55,2 \pm 12,30$ & $68,94 \pm 13,07$ & $73,53 \pm 15,53$ & $71,58 \pm 10,72$ \\
\hline & \multicolumn{2}{|c|}{$\mathrm{p}=0,13$} & \multicolumn{2}{|c|}{$\mathrm{p}=1,00$} \\
\hline \multirow{2}{*}{$\% \mathrm{G}$} & $22,88 \pm 6,64$ & $18,6 \pm 6,57$ & $26,66 \pm 6,49$ & $19,62 \pm 7,04$ \\
\hline & \multicolumn{2}{|c|}{$\mathrm{p}=0,36$} & \multicolumn{2}{|c|}{$\mathrm{p}=0,01$} \\
\hline \multirow{2}{*}{ RCQ } & $0,71 \pm 0,02$ & $0,78 \pm 0,07$ & $0,79 \pm 0,05$ & $0,79 \pm 0,05$ \\
\hline & \multicolumn{2}{|c|}{$\mathrm{p}=0,14$} & \multicolumn{2}{|c|}{$\mathrm{p}=0,87$} \\
\hline \multirow{2}{*}{ IMC } & $22,5 \pm 3,11$ & $23,4 \pm 2,88$ & $26,03 \pm 3,46$ & $24,45 \pm 3,47$ \\
\hline & \multicolumn{2}{|c|}{$\mathrm{p}=0,65$} & \multicolumn{2}{|c|}{$\mathrm{p}=0,32$} \\
\hline \multirow{2}{*}{$\mathrm{MM}(\mathrm{Kg})$} & $43,38 \pm 4,08$ & $56,43 \pm 12,91$ & $53,75 \pm 11,54$ & $57,23 \pm 8,22$ \\
\hline & \multicolumn{2}{|c|}{$\mathrm{p}=0,19$} & \multicolumn{2}{|c|}{$\mathrm{p}=0,35$} \\
\hline \multirow{2}{*}{ MG (Kg) } & $13,31 \pm 6,09$ & $12,5 \pm 4,36$ & $19,77 \pm 7,13$ & $14,32 \pm 6,39$ \\
\hline & \multicolumn{2}{|c|}{$\mathrm{p}=0,54$} & \multicolumn{2}{|c|}{$\mathrm{p}=0,04$} \\
\hline
\end{tabular}

Com relação à faixa etária, verificou-se que os aderentes e desistentes de mais de 30 anos apresentaram maior percentual de gordura e massa gorda do que os de até 30 anos. Ainda, os aderentes de até 30 anos apresentaram maior massa magra do que os de mais de 30 anos (Tabela 3).
Quando se compararam os aderentes e desistentes do TF acima de 30 anos, verificouse que os desistentes apresentaram maiores peso $(\mathrm{p}=0,04)$ e IMC $(\mathrm{p}=0,02)$ do que os aderentes; por outro lado, eles não apresentaram diferenças significativas em nenhuma das variáveis antropométricas investigadas ( $p>0,05)$. 
Tabela 3 - Análise comparativa das características antropométricas de aderentes e desistentes de TF pertencentes a diferentes faixas etárias $(\dot{x} / \pm)$.

\begin{tabular}{|c|c|c|c|c|}
\hline & \multicolumn{2}{|c|}{ Aderentes } & \multicolumn{2}{|c|}{ Desistentes } \\
\hline & Abaixo de 30 & Acima de 30 & Abaixo de 30 & Acima de 30 \\
\hline \multirow{2}{*}{ Peso $(\mathrm{Kg})$} & $71,85 \pm 14,00$ & $61,84 \pm 10,07$ & $70,50 \pm 11,04$ & $74,57 \pm 14,28$ \\
\hline & \multicolumn{2}{|c|}{$\mathrm{p}=0,08$} & \multicolumn{2}{|c|}{$\mathrm{p}=0,52$} \\
\hline \multirow{2}{*}{$\% \mathrm{G}$} & $16,16 \pm 6,04$ & $23,11 \pm 5,03$ & $18,17 \pm 6,17$ & $27,26 \pm 6,03$ \\
\hline & & & \multicolumn{2}{|c|}{$\mathrm{p}<0,01$} \\
\hline \multirow{2}{*}{ RCQ } & $0,78 \pm 0,04$ & $0,76 \pm 0,09$ & $0,79 \pm 0,05$ & $0,79 \pm 0,08$ \\
\hline & & & \multicolumn{2}{|c|}{$\mathrm{p}=0,89$} \\
\hline \multirow{2}{*}{ IMC } & $23,84 \pm 3,07$ & $22,56 \pm 2,40$ & $23,96 \pm 3,55$ & $26,38 \pm 3,04$ \\
\hline & & & \multicolumn{2}{|c|}{$\mathrm{p}=0,07$} \\
\hline \multirow{2}{*}{$\mathrm{MM}(\mathrm{Kg})$} & $60,45 \pm 13,08$ & $47,79 \pm 8,45$ & $57,33 \pm 8,19$ & $54,18 \pm 11,13$ \\
\hline & & & \multicolumn{2}{|c|}{$\mathrm{p}=0,44$} \\
\hline \multirow{2}{*}{$\mathrm{MG}(\mathrm{Kg})$} & $11,38 \pm 4,57$ & $14,34 \pm 3,53$ & $13,04 \pm 5,91$ & $20,40 \pm 6,43$ \\
\hline & & & \multicolumn{2}{|c|}{$\mathrm{p}=<0,01$} \\
\hline
\end{tabular}

Legenda: \% G = percentual de gordura; $\mathrm{RCQ}$ = razão cinturaquadril; $\mathrm{IMC}=$ índice de massa corporal; $\mathrm{MM}$ = massa magra; $\mathrm{MG}=$ massa gorda.

Foram verificadas correlaçóes positivas e significativas entre a idade e o percentual de gordura e massa gorda tanto dos aderentes quanto dos desistentes do TF. Entre os desistentes, a idade se correlacionou positivamente ao IMC. Foi verificada correlação negativa entre a idade e a massa magra dos aderentes ao TF (Tabela 4).
De modo geral, esses resultados indicam que, com o aumento da idade, ocorrem diminuição da massa magra e aumento do IMC, do percentual de gordura e da massa gorda.

Tabela 4 - Correlação das características antropométricas em função da idade dos aderentes e desistentes de TF ( $\dot{x} / \pm)$.

\begin{tabular}{|c|c|c|c|c|c|c|c|c|c|c|c|c|}
\hline \multicolumn{13}{|c|}{ Aderentes } \\
\hline & \multicolumn{2}{|c|}{ Peso } & \multicolumn{2}{|c|}{ IMC } & \multicolumn{2}{|c|}{ RCQ } & \multicolumn{2}{|c|}{$\% \mathrm{G}$} & \multicolumn{2}{|c|}{ MM } & \multicolumn{2}{|c|}{ MG } \\
\hline & $\mathrm{r}$ & $\mathrm{p}$ & $\mathrm{R}$ & $\mathrm{p}$ & $\mathrm{r}$ & $\mathrm{p}$ & $\mathrm{r}$ & $\mathrm{p}$ & r & $\mathrm{p}$ & $\mathrm{r}$ & P \\
\hline \multirow[t]{4}{*}{ Idade } & $-0,32$ & 0,11 & $-0,20$ & 0,32 & 0,00 & 0,96 & $0,57^{* *}$ & $<0,01$ & $-0,47^{*}$ & 0,01 & $0,41^{*}$ & 0,04 \\
\hline & \multicolumn{12}{|c|}{ Desistentes } \\
\hline & \multicolumn{2}{|c|}{ Peso } & \multicolumn{2}{|c|}{ IMC } & \multicolumn{2}{|c|}{ RCQ } & \multicolumn{2}{|c|}{$\% G$} & \multicolumn{2}{|c|}{ MM } & \multicolumn{2}{|c|}{ MG } \\
\hline & $\mathrm{r}$ & $\mathrm{p}$ & $\mathrm{R}$ & $\mathrm{p}$ & $\mathrm{r}$ & $\mathrm{p}$ & r & $\mathrm{p}$ & $\mathrm{r}$ & $\mathrm{p}$ & r & $\mathrm{P}$ \\
\hline Idade & 0,19 & 0,32 & $0,47^{* *}$ & 0,01 & 0,14 & 0,45 & $0,63^{* *}$ & $<0,01$ & $-0,17$ & 0,38 & $0,57^{* *}$ & $<0,01$ \\
\hline
\end{tabular}

\section{Discussão}

Dados reportados da literatura revelam que até 50\% das pessoas que iniciam um programa de atividade física desistem durante os primeiros seis meses ${ }^{13}$. Uma questáo a ser considerada e frequentemente observada nas academias é que os praticantes de TF que iniciam um programa de exercícios geralmente desejam a obtenção de resultados imediatos relacionados à estética; ao perceberem que mudanças significativas na composição corporal dependem de um processo lento que necessita de autodeterminação para a continuidade da prática, eles desistem por diversos motivos ${ }^{14}$.

Com relação às características antropométricas, verificou-se a tendência de que os desistentes apresentam maiores índice de massa gorda e IMC do que os aderentes. Sobre isso, estudos revelam que indivíduos com obesidade e elevado IMC têm maiores riscos para desistência da prática ${ }^{15-17}$. Segundo Nascimento ${ }^{18}$, os aderentes confiam mais nos resultados positivos do exercício do que os desistentes, que possuem uma percepção negativa do efeito do exercício físico. 
Uma das categorias determinantes para a prática de atividade física são as variáveis demográficas como idade, sexo e estado civil ${ }^{19}$. Com relação ao sexo, nossos achados revelaram que, independente de praticar ou não $\mathrm{TF}$, os homens sáo mais pesados e possuem mais massa muscular do que as mulheres, que por sua vez apresentam maior percentual de gordura do que os homens, corroborando com a literatura ${ }^{20}$.

Os benefícios estéticos proporcionados pela prática de TF podem ser compreendidos se analisado o fato de que os homens desistentes do TF apresentaram maior percentual de gordura do que os aderentes, e as mulheres desistentes do TF apresentaram maiores peso corporal, IMC e massa magra do que as aderentes. A eficácia do treinamento de força, particularmente para o componente muscular, como aumento da massa magra, é bem reportada na literatura ${ }^{21}$.

$\mathrm{O}$ estado civil pode estar associado à aderência ao TF devido ao suporte social, uma das categorias determinantes para a prática, em que familiares e amigos exercem influência na adoçáo de comportamentos, no caso a prática regular de exercício físico. Entretanto, os resultados deste estudo apontam que a prática de TF parece não ser influenciada pelo estado civil, pois o perfil antropométrico dos participantes da pesquisa não apresentou diferença significativa entre os sujeitos aderentes que têm ou não parceiro e os sujeitos desistentes que têm ou não parceiro. Por outro lado, nossos achados revelaram que os desistentes do TF que têm parceiro apresentaram maior percentual de gordura e massa gorda do que os desistentes do TF que não têm. Sobre isso, estudos revelam que estar casado ou viver com um parceiro está negativamente relacionado à prática de exercícios ${ }^{22,23}$. Outro importante achado deste estudo foi o fato de que, nos grupos de aderentes e desistentes, os sujeitos acima de 30 anos apresentaram maior percentual de gordura e massa gorda do que os de até 30 anos, que apresentaram maior massa magra do que os outros. Esses dados corroboram com a literatura, que aponta que com o passar dos anos ocorrem diminuiçáo da massa magra e aumento da massa gorda ${ }^{24,25}$. Ainda com relação à idade, vale destacar que os desistentes do TF que têm mais de 30 anos apresentaram maiores peso e IMC do que os aderentes do TF que têm mais de 30 anos. Isso demonstra que a prática de TF pode contribuir significativamente na redução dos efeitos deletérios da idade sobre o corpo. A literatura apresenta associação entre indicadores antropométricos de obesidade e risco coronariano elevado em adultos acima de 30 anos $^{26}$, por conseguinte recomenda-se atenção à população com essa faixa etária.

Os aspectos demográficos e antropométricos dos praticantes de exercícios físicos devem ser levados em consideração no momento de planejamento de programas de exercícios, pois essas são características importantes para o processo de aderência e manutenção da prática.

\section{Conclusão}

Conclui-se que aderentes e desistentes do TF não se diferenciaram significativamente com relação aos índices antropométricos. Quanto ao estado civil foi verificado que os desistentes do TF que têm parceiro apresentaram maior percentual de gordura e massa gorda quando comparados aos que náo têm. Aderentes e desistentes acima de 30 anos apresentaram maior percentual de gordura e massa gorda do que os de até 30 anos.

\section{Agradecimentos}

Os autores agradecem à Capes, por bolsa de pesquisa de mestrado, edital 003/2015; ao CNPQ, por bolsa de iniciação científica Pibic-CNPQ, e à Universidade do Estado de Santa Catarina (Udesc), pela oportunidade do estudo.

\section{Referências}

1. International Health, Racquet \& Sportsclub Association. The IHRSA Global Report 2016: the state of the health club industry. Boston: The Association; 2016. 128 p.

2. Ahmed C, Hilton W, Pituch K. Relations of strength training to body image among a sample of female university students. J Strength Cond Res. 2002 Nov;16(4):645-8.

3. Mezzaroba PV, Ribeiro MS, Machado FA. Comparação de dois métodos de treinamento contra resistência na força, antropometria e composição corporal de mulheres jovens. Rev Bras Ciênc Mov. 2014;22(2):106-13.

4. Costa RF, Guiselini M, Fisberg M. Correlaçâo entre porcentagem de gordura e índice de massa corporal de frequentadores de academia de ginastica. Rev Bras Ciênc Mov. 2007;15(4):39-46.

5. Carvalho T, editor. Guidelines of the Brazilian Society of Sports Medicine - dietary changes, fluid replacement, food suplements and drugs: demonstration of ergogenic action and potential health risks. Rev Bras Med Esporte. 2003;9(2):57-68.

6. Filardo RD, Leite N. Perfil dos indivíduos que iniciam programas de exercícios em academias, quanto à composição corporal e aos objetivos em relação à faixa etária e sexo. Rev Bras Med Esporte. 2001;7(2):57-61.

7. Pitanga FJG. Antropometria na avaliação da obesidade abdominal e risco coronariano. Rev Bras Cineantropom Desempenho Hum. 2011;13(3):238-41.

8. Penteado EGP, Baratto I, Silva R. Comparação entre índice de massa corporal e percentual de gordura da equipe de futsal masculino do município de Guarapuava, Paraná. Rev Bras Nutr Esp. 2010;4(21):262-7. 
9. Rezende FAC, Rosado LEFPL, Franceschinni SCC, Rosado GP, Ribeir RCL. Aplicabilidade de massa corporal na avaliação da gordura corporal. Rev Bras Med Esporte. 2010;16(2):90-4.

10. Ferreira LS, Honorato D, Stulbach T, Narciso, P. Avaliação do IMC como indicativo de gordura corporal e comparação de indicadores antropométricos para determinação de risco cardiovascular em frequentadores de academia. Rev Bras Nutr Esp. 2013;7( 42):324-32.

11. Petroski EL. Desenvolvimento e validação de equaçóes generalizadas para a estimativa da densidade corporal em adultos. Santa Maria [tese]. Santa Maria (RS): Universidade Federal de Santa Maria; 1995.

12. Siri WE. Body composition from fluid spaces and density. In: Brozek J, Henschel A, editors. Techniques for measuring body composition. Washington, DC: National Academy of Science; 1961. p. 223-44.

13. Findorff MJ, Wyman, JF, Gross CR. Predictors of longterm exercise adherence in a community-based sample of older women. J Womens Health (Larchmt). 2009 Nov; 18(11):176976.

14. Liz CM, Crocetta TB, Viana MdS, Brandt R, Andrade A. Aderência à prática de exercícios físicos em academias de ginástica. Motriz: Rev Educ Fís. 2010;16(1):181-8.

15. Costa BVD, Bottcher LB, Kokubun E. Aderência a um programa de atividade física e fatores associados. Motriz: Rev Educ Fís. 2009; 15(1):25-36.

16. Mendes FDSNS, Castro CLB, Araújo CGS. Obesos apresentam menor aderência a programa de exercício supervisionado. Rev Bras Cardiol. 2010;23(4):230-7.

17. Meseguer CM, Galán I, Herruzo R, Zorrilla B, RodriguezArtalejo F. Leisure-time physical activity in a southern European mediterranean country: adherence to recommendations and determining factors. Rev Esp Cardiol. 2009 Oct;62(10):1125-33.
18. Nascimento MC, Silva OMP, Saggioratto CML, Vargas KCB, Schopf K, Klunk J. O desafio da adesão aos exercícios físicos em grupos de idosos em Palmitos/SC: motivos para a prática e para a desistência. Rev Bras Ativ Fís Saúde. 2010;15(3):140-4.

19. Pitanga FJG. Tempo de permanência em programas de exercícios físicos em hipertensos de ambos os sexos: estudo através da análise de sobrevida. Rev baiana educ fís. 2001;2(3):310.

20. Santos DM, Sichieri R. Índice de massa corporal e indicadores antropométricos de adiposidade em idosos. Rev Saúde Pública. 2005;39(2):163-8.

21. Santos CF, Crestan TA, Picheth DM, Felix G, Mattanó RS, Porto DB, et al. Efeito de 20 semanas de treinamento com pesos sobre indicadores de composiçáo corporal. Rev Bras Ciên e Mov. 2002 abr;10(2):79-84.

22. Trost SG, Owen N, Bauman AE, Sallis JF, Brown W. Correlates of adults' participation in physical activity: review and update. Med Sci Sports Exerc. 2002 Dec;34(12):1996-2001.

23. Bergman P, Grjibovski AM, Hagströmer M, Bauman A, Sjöström M. Adherence to physical activity recommendations and the influence of socio-demographic correlates: a populationbased crosssectional study. BMC Public Health. 2008;8:367-76.

24. Matsudo SM, Matsudo VKR, Barros Neto TL. Impacto do envelhecimento nas variáveis antropométricas, neuromotoras e metabólicas da aptidão física. Rev Bras Ciên e Mov. 2000;8(4):21-32.

25. Filiardo RD, Neto CSP. Indicadores antropométricos e da composição corporal de homens e mulheres entre 20 e 39, 9 anos de idade. Rev Bras Cineantropom Desempenho Hum. 2001;3(1):55-62.

26. Pitanga FJG, Lessa I. Associação entre indicadores antropométricos de obesidade e risco coronariano em adultos na cidade de Salvador, Bahia, Brasil. Rev Bras Epidemiol. 2007;10(2)-239-48.

\section{Como citar este artigo:}

Andrade A, Dominski FH, Liz CM, Correia CK. Características antropométricas de aderentes e desistentes de treinamento de força em academia. Rev. Aten. Saúde. 2016;14(50):42-48. 